\title{
Connecting dark matter particles with the primary, obscure and normal particles through implicit causality
}

\author{
Josip Śoln ${ }^{1}$
}

\author{
${ }^{1}$ JZS Phys-Tech, Vienna, Virginia 22182, USA \\ Correspondence: Josip Śoln, JZS Phys-Tech, Vienna, Virginia 22182, USA. E-mail: jurasoln@yahoo.com
}

Received: April 11, 2017

Accepted: April 26, 2017

Online Published: May 10, 2017

doi:10.5539/apr.v9n3p1

URL: https://doi.org/10.5539/apr.v9n3p1

\begin{abstract}
The primary, obscure and normal particles with respective limiting velocities $c_{1}, c_{2}$ and $c_{3}$,solutions from bicubic equation, offer comfortable venues to tackle the newly emergent dark matter particles. Particular emphasis is given to particles with velocities of $O\left(10^{-3} \mathrm{c}\right.$ ) ( with $c$ the velocity of light) and whose energies are from $1 \mathrm{eV}$ to over $100 \mathrm{GeV}$ for which the congruent parameter $z=3 \sqrt{3} \mathrm{mv^{2 }} / 2 \mathrm{E}$ assumes values of $10^{-6}$ and $10^{-7}$. At $z=10^{-6}$ with $m c^{2}=100 \mathrm{GeV}$ one can have $E=260 \mathrm{GeV}$ or with $E=1 \mathrm{eV}$ one can have $m c^{2}=0.38 \mathrm{eV}$; while at $z=10^{-7}$ with $m c^{2}=100 \mathrm{GeV}$ one can have $E=2.6 \mathrm{TeV}$ or with $E=1 \mathrm{eV}$ one can have $m c^{2}=0.038 \mathrm{eV}$. The small values of the congruent parameter $z$ allow the limiting velocities $c_{1}, c_{2}$ and $c_{3}$ as well as the resulting energy expressions be written down perturbatevly in terms of the congruent parameter $z$.

It is shown that for $m c^{2}=100 \mathrm{GeV}$ particle in the Milky Way Dark Matter Velocity Profile (Laha, 2016), the derived limiting velocities of primary, obscure and normal particles as dark matter particles are: $c_{1}=1,7 c\left(z=10^{-7}\right)$, $1.34 c, 2.15 c\left(z=10^{-6}\right) ; c_{2}= \pm i 1,7 c\left(z=10^{-7}\right), \pm i 1.34 c, \pm i 2.15 c\left(z=10^{-6}\right)$, and $c_{3}=v\left(z=10^{-7}, 10^{-6}\right)$. Perturbatively, for a very small common primary and obscure particle velocity $v$ compared to the absolute values of their limiting velocities, one shows that the obscure particle acquires $\left(-m v^{2}\right)$ intrinsic negative energy with respect to the primary particle,with $m$ being their common mass.
\end{abstract}

Keywords: Dark matter particles, Implicit causality, Prime, Obscure and Normal particle limiting velocities

\section{Introduction}

The particle limiting velocity solutions of primary $c_{1}$, obscure $c_{2}$ and normal $c_{3}$ (Soln, 2014, 2015, 2016, [1, 2, 3]), repeated bellow with $(2.0,1,2,3)$, cathegorize particles respectively into the primary, obscure and normal particles with the help of dimensionless congruent particle parameter

$$
z=\frac{3 \sqrt{3} m v^{2}}{2 E} ;-1 \leq z \leq 1
$$

where $m, v$ and $E$ are respectively particle mass, velocity and energy. For a given value of $z$, relation (1.0) indicates that $m, v$ and $E$ are in an implicit causality relation with each other,which is affecting their allowed values, depending on the specific value of $z$. Some of these may or may not change as $z$ changes from one value to another, but always respecting that $-1 \leq z \leq 1$. The values of $c_{1}, c_{2}$ and $c_{3}$ change only if the congruent parameter as a whole changes, as can be seen from relations (2) bellow. That is, the changes in $m, v$ and $E$ must be such that they change the value of $z$ within allowed limits, which in turn, will change the respective values of $c_{1}, c_{2}$ and $c_{3}$. Of course, although the fixed value of $z$ fixes the values of $c_{1}, c_{2}$ and $c_{3}$ it does not mean that all these values are observable with particles; what it means is that they are allowed to be created. Perhaps what one observes could be a particle with $c_{3}$ or particles with $c_{1}$ and $c_{2}$.

Complete description of a dark matter particle requires also the knowledge of $m, v$ and $E$ for a given value of its congruent parameter $z$. Unfortunately the attributes of dark matter particles are not very well known. The velocities of dark matter particles appear to be the easiesr to estimate. For instance, Fan, Reece and Wang (2010) as well as Bezrukov and Gorbunov (2015) found that dark matter particles with $v \approx 10^{-3} c$ (with $c$ the velocity of light) and small energy of $E \approx 1 \mathrm{eV}$ are likely to exist. From (4), that follows, one sees that with given $v$ and $E$ the maximum 
mass is achieved at $z=1$ which in Śln (2016),was treated as a test particle. Dealing with this kind of test particles,one finds that at $z=1, v \approx 10^{-3} \mathrm{c}$ and $E \approx 1 \mathrm{eV}$, the corresponding primary, obscure and normal limiting velocity self energies to be $m(1) c_{1,3}^{2} \approx 0.58 \mathrm{eV}, m(1)\left(-c_{2}^{2}\right) \approx 1.15 \mathrm{eV}$. As each of these particles has $E \approx 1 \mathrm{eV}$, the obscure particle has to go through intrinsic self-annihilation so that $m(1)\left(-c_{2}^{2}\right) \approx 1.15 \mathrm{eV}$ is sufficiently decreased so that its energy ends up with $E \approx 1 \mathrm{eV}$ as shown in Śln (2016). Here this self-annihilation phenomenon for the obscure particle is pointed up perturbatively at the end of Section 2.

One will have to move away from $z=1$ in order to be able to discuss dark matter particles with $m c^{2}$ say, from bellow $1 \mathrm{eV}$ to above $100 \mathrm{GeV}$ as advocated recently by Laha (2016), however with velocities that cover the range: $(1 / 4) 10^{-3} \leq v \leq(4 / 3) 10^{-3} c$. In fact, the congruent parameter will assume values of $z=10^{-6}$ and $10^{-7}$ for the energies, from less than $1 \mathrm{eV}$ to over $100 \mathrm{eV}$. Specifically, with $z=10^{-6}$,the implicit causality requires that for $m c^{2}=100 \mathrm{GeV}$ one has to have at least $E=260 \mathrm{GeV}$, or for $E=1 \mathrm{eV}$ one has to have at least $m c^{2}=0.38 \mathrm{eV}$; while at $z=10^{-7}$ the implicit causality demands that for $m c^{2}=100 \mathrm{GeV}$ one has to have at least $E=2.6 \mathrm{TeV}$ or with $E=1 \mathrm{eV}$ one has tohave at least $m c^{2}=0.038 \mathrm{eV}$, etc. Of course, one notices that the congruent parameters values here for possible dark matter particles are different from $z=10^{-11}$ like when calculating in Śoln $(2014,2015$, 2016) the limiting velocity for OPERA muon electron experiment from Adam et al. (2012), and the Crab Nebula Flare 2010 observation Stecker (2014) of the superluminal electron limiting velocity (Śoln, 2014, 2015, 2016).

In Section 2 one starts with exact forms of limiting velocities $c_{1}, c_{2}$ and $c_{3}$ which depend on inverse trigonometric functions and the dimensionless congruent parameter $z$ (1). At $10^{-2} \leq z \leq 1$ the exact limiting velocity forms in calculations have to be used, while at $z \leq 10^{-2}$ either exact or perturbative forms can be used in calculations, where perturbative forms are approximations from the Taylor series with the algebraic function forms in $z$. Also for small $z$, if necessary, other relevant relations involving $E, v$ and $m$ will be expressed as algebraic functions in $z$, utilizing a new established symmetry between $c_{1}$ and $c_{2}$ under reflection of $z, z \rightarrow-z$. Also in Section 2, from Śoln (2016) two different energy expressions for primary, obscure and normal particles are presented. These are then used to exhibit the self annihilating property of the obscure particle relative to the primary particle for very small particle velocity compared to respective absolute values of limiting velocities.

Section 3 is devoted to numerical results associated with proposed dark matter particle velocity $v \sim 10^{-3} c$, where $c$ is the velocity of light (Fan, Reece, \& Wang, 2010; Bezrukov \& Gorbunov, 2015; Laha, 2016). First, a general approach is given for $v \sim 10^{-3} c$ dark matter particle observability through primary and normal particles with respect to related limiting velocities $c_{1}$ and $c_{3}$ as well as through possible effects of obscure particle with imaginary limiting velocity $c_{2}$. On a more specific level, Laha,s results (Laha, 2016) on the Milky Way dark matter velocity profiles is dissected into three segments with velocity values: initial, $v=(1 / 3) 10^{-3}$, middle, $v=(2.5 / 3) 10^{-3}$, and the end, $v=(4 / 3) 10^{-3} c$. Each of these velocities is associated formally with a respective particle. These way, one can follow much easier with more precise values of the corresponding congruent parameters and energies and other things. In fact, in the calculations with these velocities $z$ is selected with implicit causality from acustomary requirement $m c^{2}<E$ plus a must requirement $-1 \leq z \leq 1$ yielding allowed values $z \lesssim 10^{-6}, 10^{-7}$. Although the requirement $m c^{2}<E$ seems to be working, so far satisfactory, out of curiosity, one should be open to possibility to replace $c$ with $c_{1}, c_{2}$ or even with $c_{3} \neq c$ to see whether that would make a difference.

\section{Particle limiting velocity expressions with different ranges of the congruent parameter $z$}

It has been shown in Śoln $(2014,2015)$ and particularly in Śln (2016) that combining the particle nass-shell condition with the particle momentum, one ends up for $c$, identified as a limiting velocity, with the bicubic equation

$$
m^{2}\left(c^{2}\right)^{3}-E^{2} c^{2}+E^{2} v^{2}=0
$$

whose three solutions, according to Śln $(2014,2015,2016)$, are squares of the primary $c_{1}$,obscure $c_{2}$, and normal $c_{3}$, limiting velocities, which with $z$ from (1.0), are written as,

$$
\begin{gathered}
D=\frac{1}{4}\left(\frac{3 \sqrt{3}}{2 z}\right)^{4}\left[1-\frac{4}{27}\left(\frac{3 \sqrt{3}}{2 z}\right)^{2}\right]=\left(\frac{3}{2}\right)^{6} \frac{1}{z^{4}}\left(1-\frac{1}{z^{2}}\right) \leq 0, \\
z=\frac{3 \sqrt{3} m v^{2}}{2 E} ;-1 \leq z \leq 1, \frac{c_{1}^{2}}{v^{2}}=\frac{3}{z} \sin \left(\frac{\pi}{3}-\frac{1}{3} \sin ^{-1}(z)\right)>0,
\end{gathered}
$$




$$
\begin{gathered}
\frac{c_{2}^{2}}{v^{2}}=-\frac{3}{z} \cos \left(\frac{1}{3} \sin ^{-1}(z)-\frac{\pi}{6}\right)<0, \\
\frac{c_{3}^{2}}{v^{2}}=\frac{3}{z} \sin \left(\frac{1}{3} \sin ^{-1}(z)\right)>0
\end{gathered}
$$

With identities, (3.0) where $\alpha$ is a real quantity, one obtains from (2. 2)

$$
\begin{gathered}
\sin \left(\alpha+\frac{\pi}{3}\right)=\cos \left(\alpha-\frac{\pi}{6}\right)=\cos \left(-\alpha+\frac{\pi}{6}\right) \\
\text { (2.2) }: \frac{c_{2}^{2}}{v^{2}}=-\frac{3}{z} \sin \left(\frac{1}{3} \sin ^{-1}(z)+\frac{\pi}{3}\right) \prec 0
\end{gathered}
$$

From comparison of $(2,1)$ and (3.1) it is easily seen the interesting connection between $c_{1}^{2}$ and $c_{2}^{2}$ under reflection of the congruent parameter $z \rightarrow-z$, while $c_{3}^{2}$ remains the same,

$$
(2.1) \frac{c_{1}^{2}}{v^{2}}(z \rightarrow-z) \rightarrow(3.1) \frac{c_{2}^{2}}{v^{2}}(z) ;(2.3) \frac{c_{3}^{2}}{v^{2}}(z \rightarrow-z) \rightarrow(2.3) \frac{c_{3}^{2}}{v^{2}}(z)
$$

The meaning of (3.2) is imposing itself through $z$; If, for instance, the energy $E$ becomes negative in the primary particle, then the primary particle transitions into the obscure one, but treating $E$ as its positive energy. Of course, by the same token the reflection $z \rightarrow-z$ can change the obscure particle in (3.1) into the primary particle in (2.1). Now, as the normal particle is even under $z \rightarrow-z$, it simply remains the normal particle as $c_{3}^{2}$ recognizes effectively only $|z|$. Furthermore if these transitions between dark matter primary and obscure particles occur causally, one can see difficulties in pin-pointing a dark matter particle since the basic difference between primary, with real $c_{1}$, and obscure, with imaginary $c_{2}$, are in their limiting velocities.

The Taylor series expansions of $(2.1,2,3)$ ) and of (3.1) for limiting velocities in terms of $z \leq 10^{-2}$, explicitly demonstrates relations (3.2) in this approximation.

$$
\begin{gathered}
\text { (2.1) : } \frac{c_{1}^{2}}{v^{2}}(z)=\frac{3 \sqrt{3}}{2 z}-\frac{1}{2}-\frac{\sqrt{3} z}{12}-\frac{2 z^{2}}{27}+O\left(\left(z^{3}\right),\right. \\
(3.1): \frac{c_{2}^{2}}{v^{2}}(z)=-\frac{3 \sqrt{3}}{2 z}-\frac{1}{2}+\frac{\sqrt{3} z}{12}-\frac{2 z^{2}}{27}-O\left(\left(z^{3}\right),\right. \\
(2.3): \frac{c_{3}^{2}}{v^{2}}(z)=1+\frac{4 z^{2}}{27}+O\left(\left(z^{4}\right) .\right.
\end{gathered}
$$

These relations show more clearly the interrelationship between primary, obscure and normal limiting velocities $c_{1}, c_{2}$ and $c_{3}$ at small $z$ values. One notices that at small $z$ values $c_{3}^{2} \simeq v^{2}$ while the same is not true for either $c_{1}^{2}$ or $c_{2}^{2}$.

The zero square sum rule of limiting velocities (Śoln, 2014, 2015, 2015), written here as $c_{3}^{2}(z)=-c_{1}^{2}(z)-c_{2}^{2}(z)$ and valid for any congruent parameter $z$ value, shows deep interrelationship between $c_{1}^{2}, c_{2}^{2}$ and $c_{3}^{2}$. Here, of course this is explicitly seen for $z \leq 10^{-2}$ from $(4.1,2,3)$. However, the perturbation relations (4) will be very useful in evaluating ranges of limiting velocities when $v \sim 10^{-3} c$ as advocated in Fan, Reece and Wang (2010), Bezukov and Gorbunov (2015) and more recently by B. Laha in Laha (2016).

For the sake of completeness, according to Śln (2016) one writes down two different energy expressions for each of primary, obscure and normal particle whose respective limiting velocities satisfy, $c_{1}^{2}>0, c_{2}^{2} \prec 0$ and $c_{3}^{2}>0$,

$$
\begin{gathered}
E\left(c_{1}\right)=\frac{3 \sqrt{3} m v^{2}}{2 z}=\frac{\sqrt{3} m c_{1}^{2}}{2 \sin \left[\frac{1}{3}\left(\pi-\sin ^{-1}(z)\right)\right]}=m c_{1}^{2}\left(1-\frac{v^{2}}{c_{1}^{2}}\right)^{-\frac{1}{2}} \\
=m c_{1}^{2}+\frac{m v^{2}}{2}+\frac{3}{8} m c_{1}^{2}\left(\frac{v^{2}}{c_{1}^{2}}\right)^{2}+m c_{1}^{2} O\left[\left(\frac{v^{2}}{c_{1}^{2}}\right)^{3}\right]
\end{gathered}
$$




$$
\begin{gathered}
E\left(c_{2}\right)=\frac{3 \sqrt{3} m v^{2}}{2 z}=\frac{\sqrt{3} m\left(-c_{2}^{2}\right)}{2 \sin \left[\frac{1}{3} \sin ^{-1}(z)+\frac{\pi}{3}\right]}=m\left(-c_{2}^{2}\right)\left(1+\frac{v^{2}}{\left(-c_{2}^{2}\right)}\right)^{-\frac{1}{2}} \\
=m\left(-c_{2}^{2}\right)-\frac{m v^{2}}{2}+\frac{3}{8} m\left|c_{2}^{2}\right|\left(\frac{v^{2}}{\left|c_{2}^{2}\right|}\right)^{2}+m\left|c_{2}^{2}\right| O\left[\left(\frac{v^{2}}{\left|c_{2}^{2}\right|}\right)^{3}\right], \\
E\left(c_{3}\right)=\frac{3 \sqrt{3} m v^{2}}{2 z}=\frac{\sqrt{3} m c_{3}^{2}}{2 \sin \left[\frac{1}{3} \sin ^{-1}(z)\right]}=m c_{3}^{2}\left(1-\frac{v^{2}}{c_{3}^{2}}\right)^{-\frac{1}{2}} \\
=m c_{3}^{2}+\frac{m v^{2}}{2}+\frac{3}{8} m c_{3}^{2}\left(\frac{v^{2}}{c_{3}^{2}}\right)^{2}+\ldots
\end{gathered}
$$

The series expansion for $E\left(c_{3}\right)$ is not saturated, indicating slow convergence. Subtracting (5.3) from (5.6) and taking into account from the Table that $c_{1}^{2} \approx-c_{2}^{2}$, then with the same mass $m$, same $v$, same $z$, one obtains perturbatively, generally very small difference between $E\left(c_{1}\right)$ and $E\left(c_{2}\right)$,

$$
E\left(c_{2}\right)-E\left(c_{1}\right)=-m v^{2}+m\left|c_{2}^{2}\right| O\left[\left(\frac{v^{2}}{\left|c_{2}^{2}\right|}\right)^{3}\right]-m c_{1}^{2} O\left[\left(\frac{v^{2}}{c_{1}^{2}}\right)^{3}\right] \approx-m v^{2}
$$

Relation (.2) indicates that globally $E\left(c_{2}\right)$ relative to $E\left(c_{1}\right)$ exhibits self annihilation properties of the obscure particle relative to the primary particle. Presently, at very low $z=10^{-7}$ with $m v^{2} \ll m c_{1}^{2}, m\left(-c_{2}^{2}\right)$, then $\left(-m v^{2}\right)$ is rather small compared to $E\left(c_{2}\right.$ and $E\left(c_{1}\right)$. The importance of (5.10) is in the fact that the negative relative energy $E\left(c_{2}\right)-E\left(c_{1}\right)$ under the circumstances of very large congruent parameter $z$, may become even more negative indicating deeper physical differences between obscure and primary particle

\section{Limiting velocities for (dark matter) particles with small ordinary velocities}

A number of authors, such as Fan, Reece, and Wang (2010), Bezrukov and Gorbunov (2015) and Laha (2016) believe that an ordinary velocity of $v \sim 10^{-3} c$ would be a natural representative velocity for a bunch of dark matter particles, either with small mass and energy, $m c^{2} \leq E \leq 1 \mathrm{eV}$ (Fan, Reece, \& Wang, 2010; Bezukov \& Gorbunov, 2015) or with large energy and mass, $E \geq m c^{2} \geq 100 \mathrm{GeV}$ (Laha, 2016). These relatively small velocities of $O\left(10^{-3} c\right)$ facilitate bunching of these particles and their observation.

The Milky Way Dark Matter Velocity Profiles can be cast in a variety of VDF's (Velocity Distribution Functions) (Laha, 2016) of which the simplest is the one like the standard Maxwellian distribution (Laha, 2016),

$$
f(v)=A \exp \left[-\left(\frac{v}{v_{0}}\right)^{2}\right], v=|\vec{v}|
$$

The VDF $f(v)$ has maximum at $v_{0}$, and $v$ is significantly different from 0 between $v_{m n}$ and $v_{m x}$, with numerical values as follows,

$$
\begin{gathered}
k m s^{-1}=(1 / 3) 10^{-3} c: v_{m n}=0 k m s^{-1}=0 c \\
v_{0}=250{k m s^{-1}}^{-1}=(5 / 6) 10^{-3} c, v_{m x}=500 k m s^{-1}=\left(\frac{4}{3}\right) 10^{-3} c
\end{gathered}
$$

The constant $A$ in the Dark Matter Velocity Profile is the normalization factor chosen such that the intergral

$$
\int_{v_{m n}}^{v_{m x}} d^{3} v f(v)=4 \pi \int_{v_{m n}}^{v_{m x}} v^{2} d v f(v)
$$

equals the number of dark matter particles in a region of interest (Mao et al., 2013).

An important thing that this Milky Way Dark Matter Velocity Profile offers is a number of particles with velocities that are close to $10^{-3} \mathrm{c}$ and which kinematically, through primary, obscure and normal particles, could shed important light on the nature of dark matter particles. To this end, the choice of three different ordinary velocities 
between $v_{m n}$ and $v_{m x}$ are assigned to three hypothetical dark matter particles from which then the corresponding primary, obscure or normal limiting velocities are to be calculated,

$$
\begin{gathered}
v=100 \mathrm{kms}^{-1}=\frac{1}{3} 10^{-3} \mathrm{c}, \\
v=v_{0}=250 \mathrm{kms}^{-1}=\frac{5}{6} 10^{-3} \mathrm{c}, \\
v=400 \mathrm{kms}^{-1}=\frac{4}{3} 10^{-3} \mathrm{c}
\end{gathered}
$$

The question now is: What kind of limiting velocities one can expect from ordinary velocities from $(6.2,3,4) ?$ In order to use them in the implicit causality relations, one first combines $m c^{2} / E, \leq 1$ with $0 \leq z<1$ to obtain

$$
\begin{gathered}
z=\frac{3 \sqrt{3} v^{2}}{2 c^{2}} \frac{m c^{2}}{E} \leq 1 ; \frac{m c^{2}}{E} \leq 1 ; \\
\frac{2 c^{2}}{3 \sqrt{3} v^{2}} z \leq 1: z \leq \frac{3 \sqrt{3} v^{2}}{2 c^{2}}
\end{gathered}
$$

Since the ordinary velocities are assigned, one simply applies the implicit causality on (7. 4) in order to deduce the most appropriate values for $z$.

$$
\begin{gathered}
v=\frac{1}{3} 10^{-3} c, z<\frac{3 \sqrt{3}}{2 \cdot 9} 10^{-6}=0.29 \cdot 10^{-6} ; z \sim 10^{-7} \\
v=\frac{5}{6} 10^{-3} c, z<\frac{3 \sqrt{3}}{2}\left(\frac{5}{6}\right)^{2} 10^{-6}=1.8 \cdot 10^{-6} ; z \sim 10^{-6} \\
v=\frac{4}{3} 10^{-3} c, z<\frac{3 \sqrt{3}}{2}\left(\frac{4}{3}\right)^{2} 10^{-6}=4.62 \cdot 10^{-6} ; z \sim 10^{-6}
\end{gathered}
$$

In relations $(7.5,6,7)$ the choices of $z \sim 10^{-7}$ and $z \sim 10^{-6}$ are made with the largest $z^{\prime} s$ that comfortably satisfy $(7.5,6,7)$ for each particular $v$. As such they define models that likely will describe the realistic physics of the possible primary, obscure or even normal dark matter particles. The choices of $z \sim 10^{-7}, 10^{-6}$ cover cases from references (Fan, Reece, \& Wang, 2010; Bezukov \& Gorbunov, 2015; Laha, 2016) as long as $m c^{2} \leq E$, despite the fact that in Fan, Reece, and Wang (2010) and Bezukov and Gorbunov (2015), $E \sim 1 \mathrm{eV}$, while in Laha (2016) $E \geq 100 \mathrm{GeV}$. It is interesting to compare for these assumed dark matter particles their congruent parameter values of $z \sim 10^{-7}, 10^{-6}$ with $z \sim 10^{-11}$ of the OPERA muon-neutrino velocity experiment (Adam et al., 2012) as shown in Śoln (2016), as well as, with $z \sim 10^{-10}$ of the Crab Nebula Flare 2010 observation of the superluminal electron velocity (Adam et al., 2012) as shown in Śoln $(2014,2015,2016)$. In both of these experiments, $v \sim O(c)$ while in present cases $v \sim O\left(10^{-3} \mathrm{c}\right)$, lowering $z$ from $10^{-10}$ or $10^{-11}$ to $10^{-6}$ or $10^{-7}$.

Now, because $z \sim 10^{-6}, 10^{-7} \ll 1$, in place of exact limiting velocity solutions (2), one can use the small congruent parameter $z$ limiting velocity solution expressions $(4,1,2,3)$ to $O\left(z^{0}\right)$. Next, the three limiting velocities $c_{1}, c_{2}$ and $c_{3}$, calculations are done within three respective $(z, v)$ combinations $(7.5,6,7)$ according to $(4,1,2,3)$. Furthermore, consistent with Laha (2016), the mass value of $m c^{2} \approx 100 \mathrm{GeV}$ is assumed. Then as seen from exact energy expressions $(5.1,4,7)$ (compare with Śln, 2016) the calculated energy expressions satisfy, $E\left(c_{1}, z\right)=E\left(c_{2}, z\right)=$ $E\left(c_{3} z\right)$. Limiting velocities with the energies are presened in two tables that follow.

In the Table terms with $v^{2} / c$ are negligible for cases from relations (7). They are here for the sake of completeness and if it is desired to increase v's to higher values. Each normal limiting velocity $c_{3}$, as relation (4.3) indicates is for the values of $z=10^{-6}$ and $10^{-7}$, just slightly larger than $v$, which is the reason for keeping the same value as $v$. In the Table a velocity $v$ can be understood as a velocity of just created or instantaneously interacting particle; which is the reason that in such situations real $v$ goes with every particle.

The energy $E$, calculated from non-perturbative relations (5.1), (5.4) and (5.7) show the same value for each $c_{1}, c_{2}$ and $c_{3}$ with fixed values of $v$ and $z$. What one sees here is that after the creation or engagement a particle becomes free and assumes limiting velocity, real $c_{1}$ for the primary particle, imaginary $c_{2}$ for the obscure particle and real 
$c_{3}$ for the normal particle. These limiting velocity values are not universal but rather reflect from which specific $v$ value, that is to say, $z$ value they started.

As shown in Śoln (2016), the energy of every kind of particle, primary, obscure or normal particle is globally governed with implicit causality from the congruent parameter $z$ through the expression (1), $E=3 \sqrt{3} m v^{2} / 2 z$. This expression changes into forms with which one emphasizes differences between primary, obscure and normal particles through respective limiting velocities $c_{1}, c_{2}$ and $c_{3}$. Globally, primary, obscure and normal particles with the same $m, v$ and $z$ will have the same energy. For instance, a difference for the obscure particle is that perturbativelly the lower order terms of energy expression will yield negative contribution as compared to the primary particle, which is due to the fact that the obscure limiting velocity $c_{2}$ is imaginary.

Tables 1. Limiting velocities and energies of dark matter particles of selected model velocities from the Milky Way Dark Matter Velocity Profile (Laha, 2016)

$$
\begin{aligned}
& \left(\begin{array}{ccc}
z: & 10^{-7}, & 10^{-6} \\
v: & \frac{1}{3} 10^{-3} c, & \frac{5}{6} 10^{-3} c \\
c_{1}: & 1.7 c-0.15\left(v^{2} / c\right), & 1.34 c-0.19\left(v^{2} / c\right) \\
c_{2} & \pm i\left[1.7 c+0.15\left(v^{2} / c\right)\right], & \pm i\left[1.34 c+0.19\left(v^{2} / c\right)\right] \\
c_{3} & \frac{1}{3} 10^{-3} c, & \frac{5}{6} 10^{-3} c \\
E / G e V & 289, & 180
\end{array}\right) \\
& \left(\begin{array}{cc}
z: & 10^{-6} \\
v: & \frac{4}{3} 10^{-3} c \\
c_{1}: & 2.15 c-0.12\left(v^{2} / c\right) \\
c_{2} & \pm i\left[2.15 c+0.12\left(v^{2} / c\right)\right] \\
c_{3} & \frac{4}{3} 10^{-3} c \\
E / G e V & 462
\end{array}\right)
\end{aligned}
$$

\section{Conclusion}

Three particle limiting velocities $c_{1}, c_{2}$ and $c_{3}$ either in the original analytical forms (Śoln, 2014, 2015, 2016) or in the present perturbative forms for very small congruent parameters $z \sim 10^{-6}, 10^{-7}$, suggest that the corresponding primary $\left(c_{1}\right)$,obscure $\left(c_{2}\right)$ and normal $\left(c_{3}\right)$ particles be good candidates for dark matter particles for the velocities of $O\left(10^{-3} \mathrm{c}\right)$ and energies from $1 \mathrm{eV}$ through $100 \mathrm{GeV}$. These facts agree with evaluations and analyses of Fan, Reece, and Wang (2010), Bezrukov and Gordunov (2015) and Laha (2016) with his formulation of the Milky Way Dark Matter Velocity Profiles. The analysis consists of casting these Profiles in a variety of VDF's from which, as here pursued, one could extract "dark matter particles" with velocities of $O\left(10^{-3} c\right)$ as it was done here.

\section{References}

Adam, T., Agafonova, N., Aleksandrov, A., Altinok, O., Sanchez, P. A., Anokhina, A., ... \& Badertscher, A. (2012). Measurement of the neutrino velocity with the OPERA detector in the CNGS beam. Journal of High Energy Physics, 2012(10), 1-37.

Bezukov, F., \& Gorbunov, D. (2015). On the applicability of approximations used in calculation of spectrum of dark matter particles produced in particle decays.

Fan, J., Reece, M., \& Wang, L. T. (2010). Non-relativistic effective theory of dark matter direct detection. Journal of Cosmology and Astroparticle Physics, 2010(11), 042.

Śoln, J. (2014). Theoretical particle limiting velocity from the bicubic equation: Neutrino example. Physics Essays, 27(3), 448-453.

Śoln, J. (2015). Particle limiting velocities from the bicubic equation derived from Einstein's kinematics: PeV electron case. Applied Physics Research, 7(4), 37.

Śoln, J. (2016). Limiting Velocities of Primary, Obscure and Normal Particles: Self-Annihilating Obscure Particle as an Example of Dark Matter Particle. Applied Physics Research, 8(5), 1.

Laha, R. (2016). The effect of dark matter velocity profile on directional detection of dark matter.

Mao, Y. Y., Strigari, L. E., Wechsler, R. H., Wu, H. Y., Hahn, O. (2013). Halo-to-halo similarity and scatter in the velocity distribution of dark matter. The Astrophysical Journal, 764(1), 35. 
Stecker, F. W. (2014). Limiting superluminal electron and neutrino velocities using the 2010 Crab Nebula flare and the IceCube PeV neutrino events. Astroparticle Physics, 56, 16-18.

\section{Copyrights}

Copyright for this article is retained by the author(s), with first publication rights granted to the journal.

This is an open-access article distributed under the terms and conditions of the Creative Commons Attribution license (http://creativecommons.org/licenses/by/3.0/). 